pneumonia and rhabdomyolysis and he was discharged after three weeks.

\section{Discussion}

This case illustrates some of the problems faced in the $A \& E$ department with the management of the confused uncooperative patient. A good history is not always available and full examination, in particular neurological assessment, may be very difficult. Even simple investigations present practical difficulties. In this case venepuncture presented additional difficulties because of the patient's intravenous drug abuse, and for further investigations he had to be sedated.

It is well recognised that "drunk" patients with head injuries are difficult to assess. This case shows that equal care must be taken when assessing the drug addict with confusion. The confusion must not be attributed to the effects of drugs until a full differential diagnoses has been considered. This must include infection, metabolic causes, subarachnoid haemorrhage, and head injury. Other than his altered mental status, the only abnormal examination finding was mild pyrexia. Any degree of pyrexia in the confused patient should be regarded as an absolute indication for urgent investigation, regardless of the difficulties associated with venepuncture and other procedures.
Meningococcal infection has a mortality of up to $24 \%$, ${ }^{1}$ which is increased if there is diagnostic or therapeutic delay. In $10 \%$ of cases the presentation is predominantly of septicaemia, with no clinical features of meningeal involvement. A purpuric or petechial rash may be present in only $52 \%$ of cases. ${ }^{2}$ In our patient, the diagnosis could easily have been missed, with disastrous consequences.

Intravenous drug abusers are at increased risk of infections. This is a result of a combination of factors, which include needle contamination, poor hygiene, and HIV infection. The most common infections requiring admission are cutaneous, respiratory, and endocardial, ${ }^{3}$ with streptococci and staphylococci being the most commonly isolated pathogens. ${ }^{4}$

There is no evidence for an association between intravenous drug abuse and meningococcal infection.

1 Strang JR, Pugh EJ. Meningococcal infections: reducing the case fatality by giving penicillin before admission to case fatality by giving penicilin
hospital. BMF 1992;305:141-3.

2 Cartwright K, Reilly S, White D, Stuart J. Early treatment with parenteral penicillin in meningococcal disease. $B M \mathcal{J}$ with parenteral penic

3 Beaufoy A. Infections in intravenous drug users: a two year review. Can f Infect Control 1993;8:7-9.

4 Weisse AB, Heller DR, Schimenti RJ, Montgomery RL, Kapila $R$. The febrile parenteral drug user, a prospective study in 121 patients. Am $\mathcal{F}$ Med 1993;94:274-80.

\title{
Perilunate fracture-dislocation: a continually missed injury
}

\author{
D H Sochart, P D Birdsall, A S Paul
}

\begin{abstract}
Five cases of perilunate fracturedislocation are presented in which the radiological appearances were typical but the diagnoses were initially missed. A simple systematic method of $x$ ray analysis is described.

(f Accid Emerg Med 1996;13:213-216)
\end{abstract}

Key terms: perilunate dislocation; missed diagnosis; $x$ ray interpretation

Department of

Orthopaedics, Bury General Hospital, Bury, Manchester

D H Sochart

Department of Orthopaedics, North

Tees Hospital

P D Birdsall

Department of Orthopaedics, Manchester Royal Infirmary, Manchester A S Paul

Correspondence to: Mr D H Sochart,

7 Woodlea, Walkden Road, Worsley, Manchester M28 2QJ, United Kingdom. instability. We present five cases in which the radiological appearances were typical but the diagnoses were initially missed and describe a simple systematic method of $x$ ray analysis to avoid future misinterpretation.

\section{Case reports}

CASE 1

A 32 year old left handed mechanic fell from his motorbike while returning from holiday in Holland. He sustained a hyperextension injury to his left wrist and attended the local hospital where $x$ rays were taken and he was told that he simply had a "chipped bone". He was supplied with a sling but during the crosschannel journey home his hand became increasingly painful and he developed numbness in his left thumb, index, and middle fingers. Following disembarkation he attended the nearest $A \& E$ department but again received the same instructions. On his return home he attended his local A\&E department, now 48 hours from the time of the injury. His wrist was very swollen and painful and he had loss of sensation in the median nerve distribution. Review of the initial $x$ rays (fig 1 ) showed obvious trans-styloid perilunate dislocation, and he was managed by open reduction and $\mathrm{K}$-wire stabilisation through a volar approach, to allow decompression of the 
badly traumatised median nerve. Because of the extreme degree of swelling, the skin was left open and secondary suturing was required. The K-wire and plaster cast were removed at four weeks and by nine months he had regained an $80 \%$ range of movement but still had diminished sensation at the tips of the index and middle fingers.

\section{A}

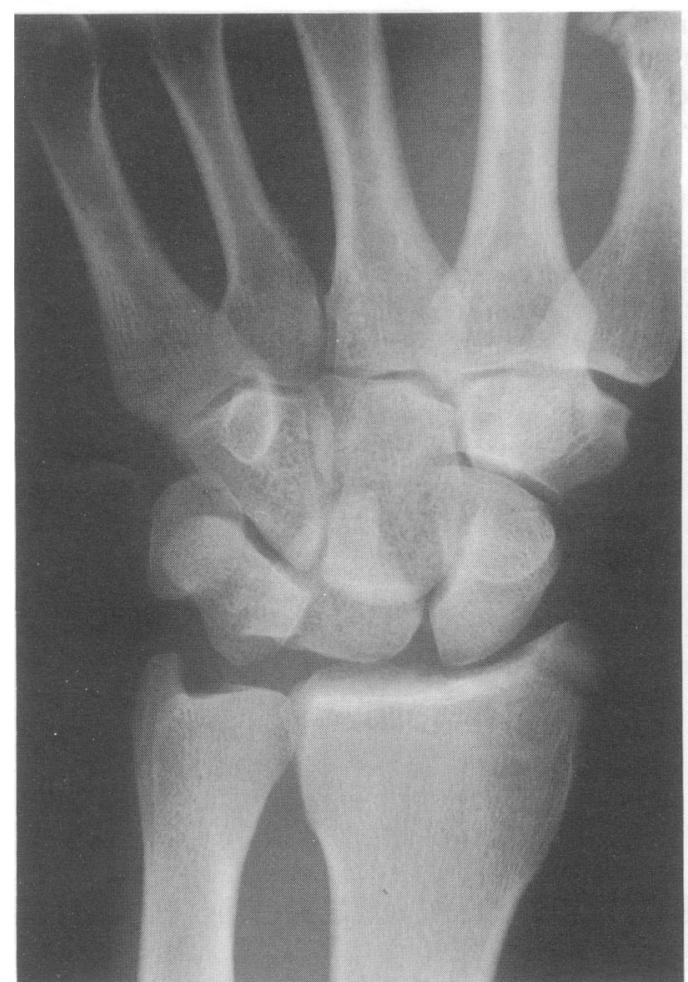

CASE 2

A 62 year old right handed woman fell down stairs three weeks after a left total hip replacement and sustained an injury to her right wrist. Radiographs were interpreted as showing a simple scaphoid fracture with some associated osteoarthritis, and she was managed conserva-

B

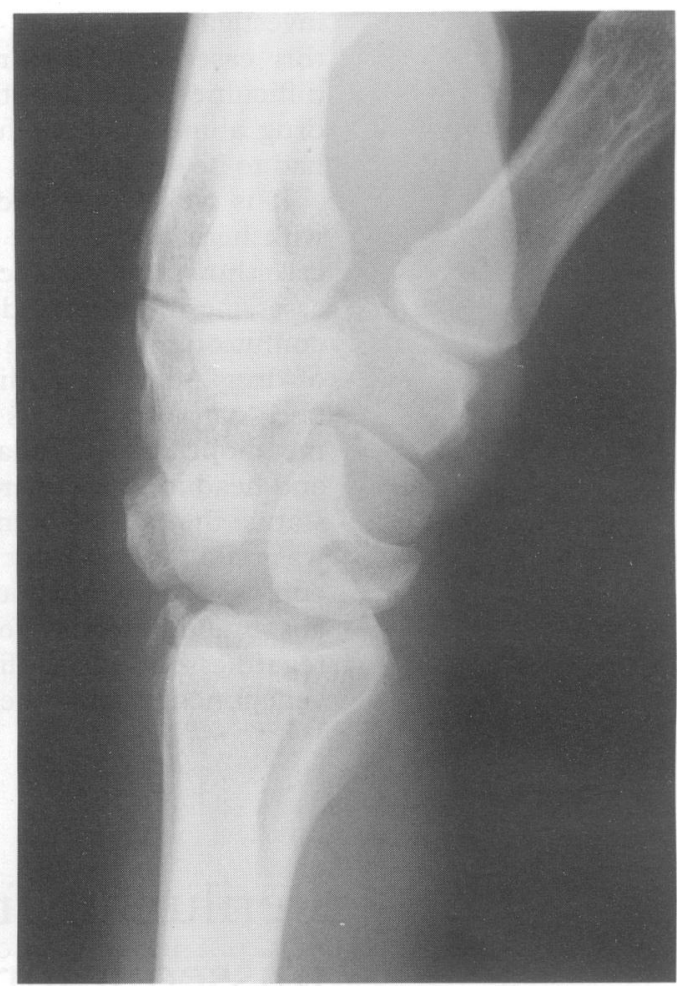

B

A

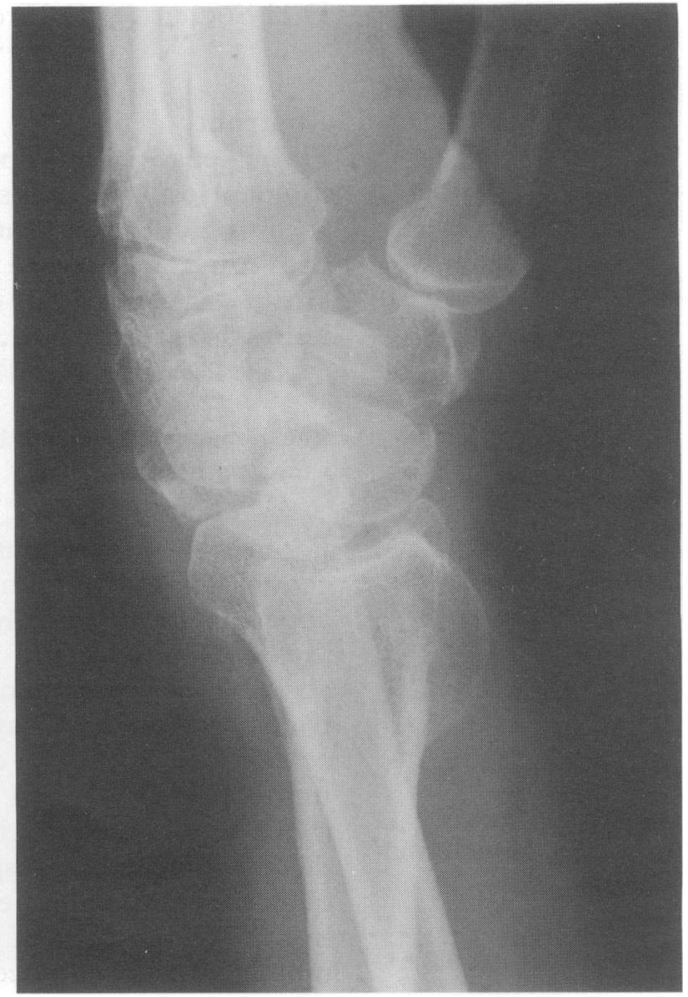


tively in a plaster cast. She developed median nerve symptoms 48 hours after the injury, and on review of the $x$ rays (fig 2) 10 days later she was confirmed to have a trans-scaphoid perilunate dislocation. Open reduction was performed through a volar approach and the bruised median nerve was decompressed, the dislocation reduced, and the scaphoid fracture stabilised with two K-wires. The median nerve symptoms fully resolved after three weeks and the wires were removed at eight weeks. She made satisfactory progress with physiotherapy and achieved a $75 \%$ range of movement.

CASE 3

A 24 year old man fell from his motorcycle while taking part in a motorcross event. $\mathrm{He}$ sustained a hyperextension injury to the right wrist and although he initially complained of numbness and paraesthesiae in the median nerve distribution these symptoms quickly resolved. Radiographs were interpreted as being normal but when the patient attended clinic a week later he was diagnosed as having a trans-scaphoid perilunate dislocation. This was manipulated under anaesthetic, with satisfactory reduction, and he was placed in a scaphoid cast. At 12 weeks he had not achieved radiological union and therefore underwent internal fixation with a Herbert screw and bone grafting from the distal radius, following which the fracture united.

CASE 4

A 40 year old man caught his right arm in heavy machinery at work. He was found to have superficial grazes on the volar aspect of his forearm and a deformed wrist but had no neurological symptoms. The initial radiographs were accepted as normal but he was diagnosed one week later as having a dorsal perilunate dislocation. He underwent closed reduction under general anaesthetic and image

A

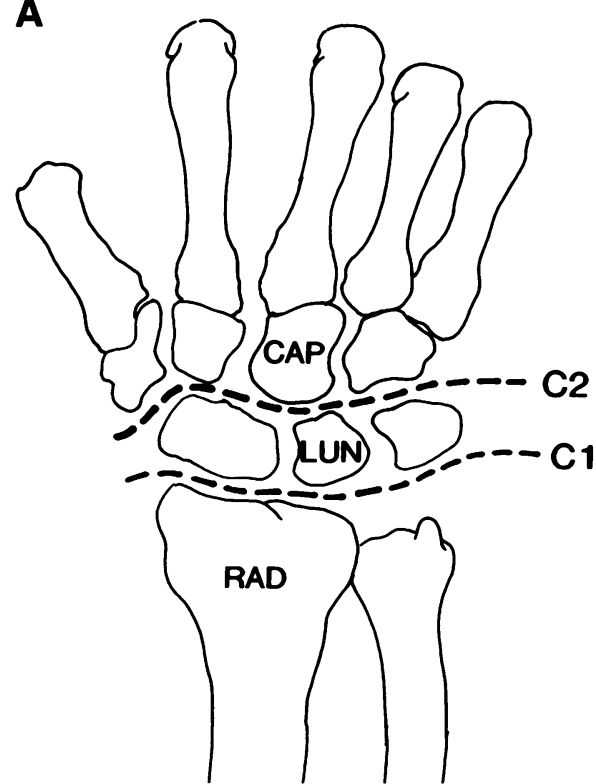

B

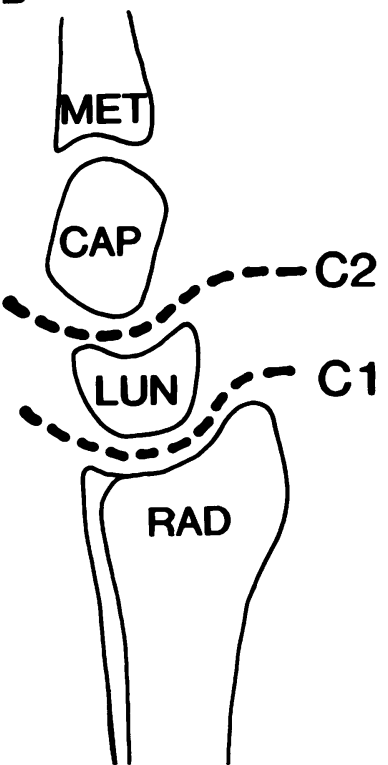

Figure $3 A P(A)$ and lateral $(B)$ diagrammatic views of wrist demonstrating curves $C 1$ and $C 2$. intensifier control and a stable reduction was achieved. A plaster cast was applied for six weeks and he subsequently obtained a full range of movement of the wrist with full functional recovery.

CASE 5

A 42 year old man was involved in a road traffic accident during which he sustained a minor head injury and an injury to the right wrist, which was very swollen and had multiple abrasions. Radiographs were taken and passed as normal, but on review three weeks later he was found to have a trans-scaphoid perilunate dislocation. He underwent open reduction and stabilisation with a Kirschner wire, which was removed five weeks later. Radiological union had occurred by six months and he achieved good wrist function but lacked 20 degrees of dorsal and palmar flexion.

\section{Discussion}

Perilunate dislocations and fracture-dislocations are rare but potentially serious wrist injuries, which are particularly important as they tend to occur in young people and may lead to considerable long term morbidity due to the development of radio-carpal arthritis, carpal instability, or median nerve damage. They were first described by Barton in $1838^{1}$ but continue to be commonly missed on initial presentation at the $A \& E$ department, being mistaken for a "simple wrist sprain". In a recent series, $25 \%$ of cases were missed, and definitive treatment was received within the first week by only $61 \%{ }^{2}$

The presence of localised swelling and deformity at the level of the radio-carpal joint are typical and there may be associated median nerve symptoms. These clinical findings, allied to a history of hyperextension injury and the presence of styloid or scaphoid fractures, should raise the possibility of radio-carpal dislocation, and the $x$ rays should be closely scrutinised. Good quality AP and lateral views are required and these should be interpreted by picturing two parallel curves (fig 3, A and B), which are determined by the crucial relations between the lunate and the head of the capitate. On the lateral $x$ ray, the first curve (C1) passes between the reciprocal convex and concave articular surfaces of the radius and lunate and is also demonstrated on the AP diagram. An $x$ ray of trans-styloid fracturedislocation of the wrist (fig 4) shows clearly the disruption of this primary curve on both views. The second curve (C2) passes between the cup shaped lunate and the head of the capitate on the lateral view and through the mid-carpal joint on the AP view. This curve is clearly disrupted following perilunate dislocation and fracture-dislocations, and systematic analysis of the $x$ rays in this manner will allow easy identification of the injury even in an arthritic wrist (fig 2).

In this series of five cases (table) there was an average delay in diagnosis and implementation of definitive treatment of nine days, and three of the patients developed symptoms and signs of median nerve compression. In all five 
A

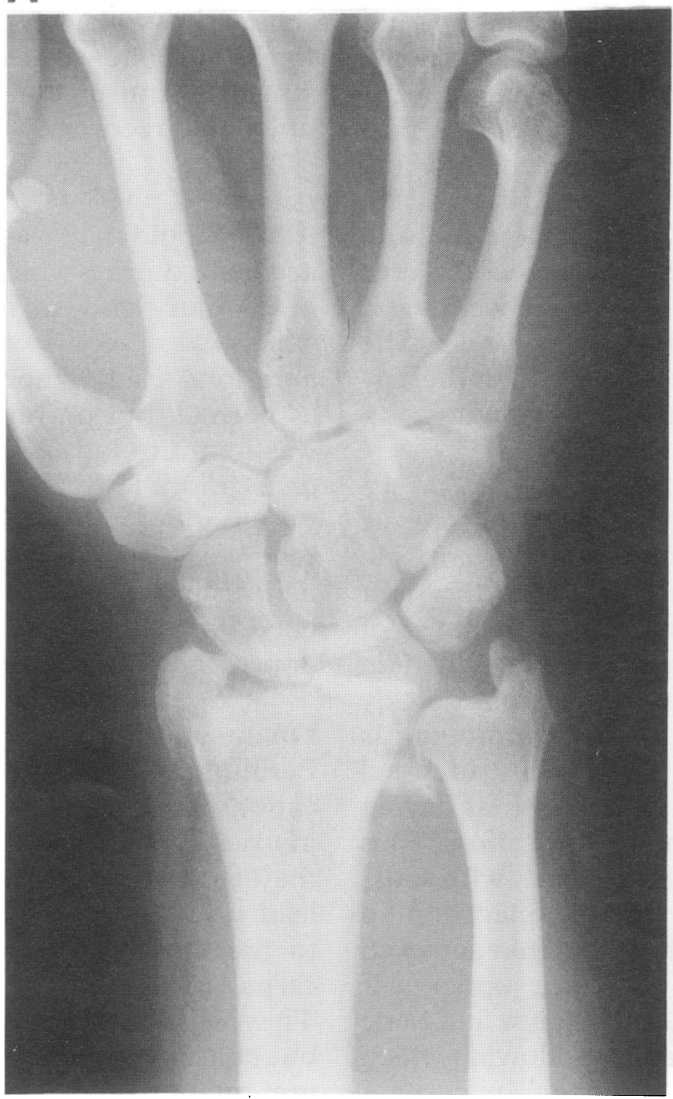

B

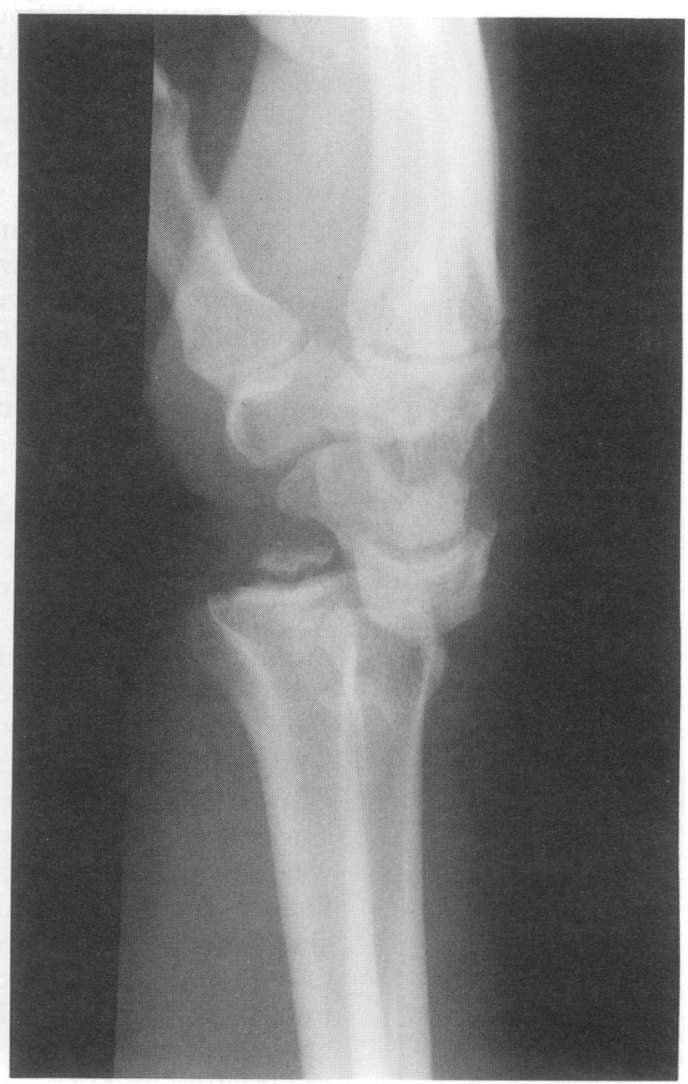

Figure $4 A P(A)$ and lateral $(B)$ views of right wrist showing trans-styloid radio-carpal fracture-dislocation.

Summary of the five reported cases

\begin{tabular}{lllllr}
\hline Patient & Age & Sex & Side & Diagnosis & Delay in treatment \\
\hline 1 & 32 & M & Left & Trans-styloid perilunate dislocation & 2 days \\
2 & 62 & F & Right & Trans-scaphoid perilunate dislocation & 10 days \\
3 & 24 & M & Right & Trans-scaphoid perilunate dislocation & 7 days \\
4 & 40 & M & Right & Perilunate dislocation & days \\
5 & 42 & M & Right & Trans-scaphoid perilunate dislocation & 21 days \\
\hline
\end{tabular}

cases the characteristic radiological appearances were initially missed by the A\&E staff. There was no routine reporting of trauma $x$ rays by radiologists on patients referred for orthopaedic follow up, and two of the cases were also missed on first attendance at the orthopaedic clinic. Early accurate diagnosis spares the patient unnecessary discomfort and allows the best chance of avoidance of median nerve dysfunction. ${ }^{3}$ It also permits conservative management when appropriate, but delay necessitates operative intervention and may compromise eventual functional outcome $\mathrm{e}^{4}$ and initiate the spiral towards late salvage surgery. We therefore recommend this method of analysis of all wrist $x$ rays and have found it very effective in establishing the correct diagnosis. The routine reporting of all A\&E $x$ rays by an experienced radiologist may also be of value as an additional fail safe measure.

1 Barton JR. Views and treatment of an important injury to the wrist. Medical Examiner 1838;1:365.

2 Herzberg G, Comtet JJ, Linscheid RL, Amadio PC, Cooney WP, Stalder J. Perilunate dislocations and fracture dislocations: a multicenter study. $\mathcal{F}$ Hand Surg Hill NA Fractur

Clin Clin North Am 1970;1:275-84.

4 Inoue G, Tanaka Y, Nakamura R. Treatment of transscaphoid perilunate dislocations by internal fixation with the Herbert screw. $\mathcal{F}$ Hand Surg 1990;15B:449-54.
Hope Hospital,

Eccles Old Road,

Salford M6 8HD,

United Kingdom:

University Department

of Emergency

Medicine

A P. Gleeson

Division of

Orthopaedics

C Brookes

G Brydon

Correspondence to:

Mr A Gleeson

Accident and Emergency

Department, Royal Infirmary

of Edinburgh, Lauriston

Place, Edinburgh EH3 9YW.

\title{
Scapholunate instability - a spectrum of pathology
}

\author{
A P Gleeson, C Brookes, G Brydon
}

Abstract

Five cases of scapholunate instability are reported. The condition is commonly misdiagnosed in accident and emergency de- partments. The importance of a complete clinical assessment of the suspected scaphoid injury and the need to measure the scapholunate distance and the scapho- 